\title{
Generalized Parton Distributions at COMPASS
}

\author{
F. H. Heinsius \\ on behalf of the COMPASS Collaboration
}

Ruhr-Universität Bochum, Institut für Experimentalphysik I, 44780 Bochum, Germany.

\begin{abstract}
Generalized parton distributions can be probed in hard exclusive meson scattering and deeply virtual Compton scattering (DVCS). Prospects for the measurements of these processes at the COMPASS experiment at CERN will be shown. A recoil detector and additional calorimetry are planned to identify the DVCS process. Simulations for different models and a test of the recoil detector have been performed.
\end{abstract}

\section{Introduction}

The generalized parton distributions (GPD) make it possible to provide a coherent description of the nucleon. They can describe elastic form factors as well as helicity dependent and helicity independent parton density distributions at the same time. The angular momentum of the quarks inside the nucleon may be determined. The generalized parton distributions can be accessed by measuring deeply virtual Compton scattering and deeply virtual meson production processes at COMPASS. The GPDs are functions of 3 parameters: the longitudinal quark momentum fraction $x$, the longitudinal momentum transfer $2 \xi$ with $\xi=x_{B j} /\left(2-x_{B j}\right)$ and the momentum transfer squared to the target nucleon $t$. The latter is the Fourier conjugate to the transverse impact parameter $r$. A three dimensional picture of the nucleon can be obtained as a function of the parameters $r$ and $x$.

The COMPASS experiment at CERN is a facility to study QCD by structure and spectroscopy measurements. It is a perfect place to study generalized parton distributions. From 2002 onwards the experiment was operated with a polarized target and various aspects related to GPDs are studied: A complete analysis of $\rho$ production is performed. Studies of exclusive $\phi$ and $2 \pi$ production are in progress. The ratio of the GPD $E / H$ function is investigated by the measurements on a transverse polarized target. The GPD $E$ functions are related to the Sivers effect [2]. Sivers asymmetries are investigated at COMPASS with muons scattered off transverse polarized deuteron and proton targets [3].

In this paper the possible measurements of the generalized parton distributions in the years 2010 - 2015 are presented.

\section{Possible measurements}

The measurements of the generalized parton distributions will be performed with an unpolarized liquid hydrogen or liquid deuterium target surrounded by a recoil detector [4]. The CERN beamline can provide polarized muons with beam energies between 100 and $190 \mathrm{GeV}$. For the main measurements we have chosen a muon energy of $100 \mathrm{GeV}$ to maximize the muon flux and the interference effects between DVCS and Bethe Heitler. Both positive and negative muons with opposite polarization of $80 \%$ and same intensities are available. For beam charge asymmetry measurements the beam charge can be changed once a day. These measurements have the advantage that not only cross sections, e.g. amplitudes squared, but also both the real and imaginary part of the DVCS amplitudes can be determined. 
Measurements of the cross section difference of opposite charged and polarized muons give rise to the real part of the DVCS amplitude, while the cross section sum provides information on the imaginary part of the DVCS amplitude.

\section{Simulations}

Simulations of the beam charge asymmetry have been performed for the COMPASS experiment under different model assumptions on the DVCS cross section. Two models are based on double distribution parameterizations of GPDs. Model 1 assumes a dependence of $H(x, \xi, t) \propto q(x) F(t)$ [5]. Model 2 includes a correlation between $x$ and $t$. It considers fast partons in the small valence core and slow partons at larger distances: $H(x, 0, t) \propto q(x) e^{t<b_{\perp}^{2}>}=q(x) / x^{\alpha^{\prime} t}$ with $\alpha^{\prime}$ the slope of the Regge trajectory and $\left\langle b_{\perp}^{2}\right\rangle=\alpha^{\prime} \ln 1 / x$ describing the transverse extension of partons in hadronic collisions. This ansatz reproduces the chiral quark-soliton model of Ref. [6]. Two values of the $\alpha^{\prime}$ parameter where applied to the simulations: $0.8(\mathrm{GeV} / c)^{-2}$ and $1.1(\mathrm{GeV} / c)^{-2}$.

Another way to parametrize the DVCS amplitudes is the dual parameterization, where one separates the $x, \xi$ dependence from the $\xi, t$ dependence. By the decomposition into Mellin moments it is straightforward to perform QCD evolution in this model. Calculations have been done by Guzey and Teckentrup [7]. Their dual parameterization with the Regge motivated $t$-dependence were used as model 3 in the simulations for COMPASS.

With a luminosity of $1.3 \cdot 10^{32} \mathrm{~cm}^{-2} \mathrm{~s}^{-1}$ and an efficiency of $25 \%$ it is possible to split the data in six bins in $Q^{2}$ from $1.5(\mathrm{GeV} / c)^{2}$ to $7.5(\mathrm{GeV} / c)^{2}$ and 3 bins in $x_{B j}$ from 0.03 to 0.27 assuming a running time of 150 days. A separation between the different models can be seen in Fig. 1. COMPASS can provide important data in the $x_{B j}$ region of $0.03-0.27$ to constrain the models.

\section{Experimental setup}

The simulations were done for an upgraded version of the COMPASS setup at CERN. The positive and negative charged muons are scattered off a liquid hydrogen or liquid deuterium target. A $2.5 \mathrm{~m}$ long target has to be designed for the DVCS measurements. In addition a recoil detector to ensure the exclusivity is needed. The target will allow a minimal $t$ of $0.06(\mathrm{GeV} / c)^{2}$. To allow for 10 bins in $t$ a minimum time resolution of $300 \mathrm{ps}$ is needed for the recoil detector. A full size prototype of the recoil detector has been tested in the fall 2006 at the COMPASS experimental area. A time resolution of $310 \mathrm{ps}$ has been achieved.

The scattered muons are detected by the COMPASS trackers: scintillating fibers, silicon detectors, Micromegas, GEMs, drift chambers, straw tubes and MWPCs. The existing electromagnetic calorimeters cover an angle $\theta<10^{\circ}$. They need to be completed by additional calorimetry to cover angles up to 30 degrees to allow for efficient $\pi^{0}$ background rejection.

\section{Outlook}

Currently simulations are being performed and a proposal for GPD measurements at COMPASS is being prepared. In the years 2007 - 2009 a recoil detector, a liquid hydrogen target and an large angle electromagnetic calorimeter are planned to be constructed. Thus measurements of GPDs could be performed at the COMPASS experiment in the years 2010 2015. First results are expected well before possible future projects at the JLAB $12 \mathrm{GeV}$ 
upgrade [8], the FAIR project [9] and at an electron ion collider [10]. A big advantage of the COMPASS experiment is that its kinematic region covers both the valence quark and the sea quark region in terms of $x_{B j}$.

\section{References}

[1] Slides: http://indico. cern. ch/contributionDisplay .py? contribId=257\&sessionId=10\&conf Id=9499

[2] M. Burkardt and D. S. Hwan, Phys. Rev. D69 074032 (2004).

[3] COMPASS Collaboration, E.S. Ageev, et al., Nucl. Phys. B 76531 (2007);

COMPASS Collaboration, V. Yu. Alexakhin, et al., Phys. Rev. Lett. 94202002 (2005).

[4] COMPASS Collaboration, "Outline for generalized parton distribution measurements with COMPASS at CERN", CERN report, CERN-SPSC-2005-007, Jan. 2005.

[5] Vanderhaeghen et al., Phys. Rev. D60 094017 (1999).

[6] Goeke et al., Prog. Part. Nucl. Phys. 47, 401 (2001).

[7] Guzey and Teckentrup, Phys. Rev. D74 054027 (2006)

[8] Z.-E. Meziani, "Jlab $12 \mathrm{GeV}$ : Large x, Spin/Flavour and GPDs", these proceedings.

[9] B. Seitz, "PANDA at GSI: Drell-Yan, transversity, exclusive channels", these proceedings.

[10] A. Bruell, "Polarized Physics with an Electron-Ion-Collider", these proceedings. 

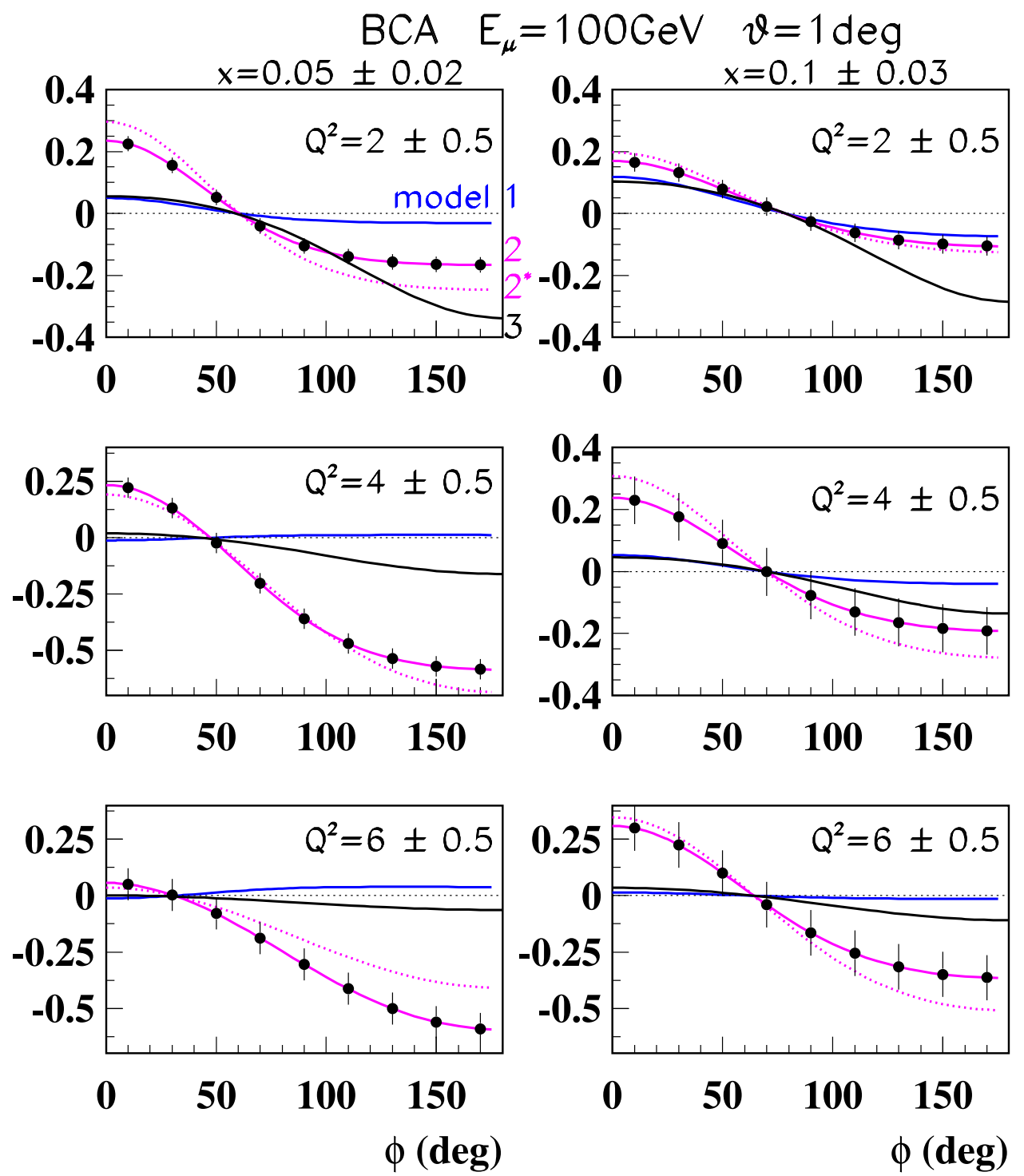

Figure 1: Azimuthal distributions of the beam charge asymmetry in 6 different $x, Q^{2}$ regions for model 1 (blue line), model 2 (line with points and error bars, $\left.\alpha^{\prime}=0.8(\mathrm{GeV} / c)^{-2}\right)$, model $2^{*}\left(\right.$ dotted, $\left.\alpha^{\prime}=1.1(\mathrm{GeV} / c)^{-2}\right)$, and model 3 (black line) as described in the text. 